\title{
Author Correction: Cycling Fermi arc electrons with Weyl orbits
}

\author{
Cheng Zhang (D), Yi Zhang, Hai-Zhou Lu, X. C. Xie and Faxian Xiu (D)
}

Correction to: Nature Reviews Physics https://doi.org/10.1038/s42254-021-00344-z, published online 15 July 2021.

In the original version of this article, the colour scheme of Fig 1 was misleading. On page 3, two equations were incorrect, using $\varepsilon$ instead of $e$ (electronic charge). The definition $k_{\mathrm{w} \|} \approx v_{\mathrm{F}} \varepsilon_{\mathrm{F}}$ was also incorrect, and should read $k_{\mathrm{F} \|} \approx v_{\mathrm{F}} \varepsilon_{\mathrm{F}}$. On page 6 , the equation $v=2\left(v_{1}+\frac{1}{2}\right)=0, \pm 3, \pm 5 \cdots$ was incorrect and should be $v=2\left(v_{1}+\frac{1}{2}\right)= \pm 1, \pm 3, \pm 5 \cdots$. These errors have now been corrected in the HTML and PDF versions of the article.

https://doi.org/10.1038/s42254-021-00378-3 | Published online 7 September 2021

(c) Springer Nature Limited 2021 